J. Korean Math. Soc. 50 (2013), No. 6, pp. 1257-1269

http://dx.doi.org/10.4134/JKMS.2013.50.6.1257

\title{
THREE SOLUTIONS FOR A CLASS OF NONLOCAL PROBLEMS IN ORLICZ-SOBOLEV SPACES
}

\author{
Nguyen Thanh Chung
}

\begin{abstract}
Using the three critical points theorem by B. Ricceri [23], we obtain a multiplicity result for a class of nonlocal problems in OrliczSobolev spaces. To our knowledge, this is the first contribution to the study of nonlocal problems in this class of functional spaces.
\end{abstract}

\section{Introduction and preliminaries}

Let $\Omega$ be a bounded domain in $\mathbb{R}^{N}(N \geq 3)$ with smooth boundary $\partial \Omega$. Assume that $a:(0, \infty) \rightarrow \mathbb{R}$ is a function such that the mapping, defined by

$$
\varphi(t):= \begin{cases}a(|t|) t & \text { for } t \neq 0, \\ 0 & \text { for } t=0,\end{cases}
$$

is an odd, increasing homeomorphism from $\mathbb{R}$ onto $\mathbb{R}$. For the function $\varphi$ above, let us define

$$
\Phi(t)=\int_{0}^{t} \varphi(s) d s \quad \text { for all } t \in \mathbb{R},
$$

on which will be imposed some suitable conditions later

In this article, we are concerned with a class of nonlocal problems in OrliczSobolev spaces of the form

$$
\left\{\begin{aligned}
-M\left(\int_{\Omega} \Phi(|\nabla u|) d x\right) \operatorname{div}(a(|\nabla u|) \nabla u) & =\lambda f(x, u)+\mu g(x, u) \text { in } \Omega, \\
u & =0 \text { on } \partial \Omega,
\end{aligned}\right.
$$

where $M: \mathbb{R}^{+} \rightarrow \mathbb{R}$ is a nondecreasing continuous function, $f, g: \Omega \times \mathbb{R} \rightarrow \mathbb{R}$ are two Carathéodory functions and $\lambda, \mu$ are two real parameters.

Received November 11, 2012; Revised July 6, 2013.

2010 Mathematics Subject Classification. 34B27, 35J60, 35B05.

Key words and phrases. nonlocal problems, Orlicz-Sobolev spaces, multiple solutions, three critical points theorem. 
It should be noticed that if $\varphi(t)=p|t|^{p-2} t$, then problem (1.1) becomes the well-known $p$-Kirchhoff type equation

$$
\left\{\begin{aligned}
-M\left(\int_{\Omega}|\nabla u|^{p} d x\right) \Delta_{p} u & =\lambda f(x, u)+\mu g(x, u) \text { in } \Omega, \\
u & =0 \text { on } \partial \Omega .
\end{aligned}\right.
$$

Since the first equation in (1.2) contains an integral over $\Omega$, it is no longer a pointwise identity, and therefore it is often called a nonlocal problem. This problem models several physical and biological systems, where $u$ describes a process which depends on the average of itself, such as the population density, see [6]. Problem (1.2) is related to the stationary version of the Kirchhoff equation

$$
\rho \frac{\partial^{2} u}{\partial t^{2}}-\left(\frac{P_{0}}{h}+\frac{E}{2 L} \int_{0}^{L}\left|\frac{\partial u}{\partial x}\right|^{2} d x\right) \frac{\partial^{2} u}{\partial x^{2}}=0
$$

presented by Kirchhoff in 1883, see [16]. This equation is an extension of the classical d'Alembert's wave equation by considering the effects of the changes in the length of the string during the vibrations. The parameters in (1.3) have the following meanings: $L$ is the length of the string, $h$ is the area of the crosssection, $E$ is the Young modulus of thematerial, $\rho$ is themass density, and $P_{0}$ is the initial tension.

In recent years, problems involving $p$-Kirchhoff type operators have been studied in many papers, we refer to $[2,5,12,18,19,24,25]$, in which the authors have used different methods to get the existence of solutions for (1.2). In the case when $p($.$) is a continuous function, problem (1.2) has also been$ studied by many authors, see for examples $[3,7,8,11,13,14]$.

We point out the fact that if $M(t) \equiv 1$ and the function $\varphi(t)$ is defined above, problem (1.1) becomes a nonlinear and non-homogeneous problem, which has been studied by some authors in Orlicz-Sobolev spaces, we refer to some recent papers $[4,9,10,15,20,21]$.

In this article, motivated by the works mentioned above, we shall study the existence of solutions for nonlocal problems of type (1.1). It is clear that this is a natural extension from the earlier studies on nonlocal problems in classical Sobolev spaces and on nonlinear non-homogeneous problems in Orlicz-Sobolev spaces. To our knowledge, this is the first contribution to the study of nonlocal problems in this class of functional spaces. More precisely, using the ideas firstly introduced in the papers $[23,24]$ and developed in $[3,4]$ we want to illustrate how to handle nonlocal problem (1.1) in Orlicz-Sobolev spaces. Moreover, motivated by the results on $p$-Kirchhoff type problems in $[3,8,12,11,14]$ we study problem (1.1) under the condition that the Kirchhoff function $M(t)$ may be degenerate in the sense that it may have value 0 at 0 . For this reason, our result in this paper is better than that of $[3,4,24]$, in which the authors consider problem (1.1) in the special cases $M(t) \equiv 1$ or $\varphi(t)=p|t|^{p-2} t$. In 
this work, we also show a mistake in the paper by F. Cammaroto et al. [4] (see Remark 2.3).

In order to study problem (1.1), let us introduce the functional spaces where it will be discussed. We will give just a brief review of some basic concepts and facts of the theory of Orlicz and Orlicz-Sobolev spaces, useful for what follows, for more details we refer the readers to the books by Adams [1], Rao and Ren [22], the papers by Clément et al. [9, 10], M. Mihăilescu et al. [20, 21] and F. Cammaroto et al. [4].

For $\varphi: \mathbb{R} \rightarrow \mathbb{R}$ and $\Phi$ introduced at the beginning of the paper, we can see that $\Phi$ is a Young function, that is, $\Phi(0)=0, \Phi$ is convex, and $\lim _{t \rightarrow+\infty} \Phi(t)=$ $+\infty$. Furthermore, $\Phi$ is an $N$-function, i.e., $\Phi$ is continuous, convex, $\Phi(t)>0$ for $t>0, \lim _{t \rightarrow 0} \frac{\Phi(t)}{t}=0$, and $\lim _{t \rightarrow+\infty} \frac{\Phi(t)}{t}=+\infty$. The function $\Phi^{*}$ defined by the formula

$$
\Phi^{*}(t)=\int_{0}^{t} \varphi^{-1}(s) d s \text { for all } t \in \mathbb{R}
$$

is called the complementary function of $\Phi$ and it satisfies the condition

$$
\Phi^{*}(t)=\sup \{s t-\Phi(s): s \geq 0\} \quad \text { for all } t \geq 0 .
$$

We observe that the function $\Phi^{*}$ is also an $N$-function in the sense above and the following Young inequality holds

$$
s t \leq \Phi(s)+\Phi^{*}(t) \quad \text { for all } s, t \geq 0 .
$$

The Orlicz class defined by the $N$-function $\Phi$ is the set

$$
K_{\Phi}(\Omega):=\left\{u: \Omega \rightarrow \mathbb{R} \text { measurable }: \int_{\Omega} \Phi(|u(x)|) d x<\infty\right\}
$$

and the Orlicz space $L_{\Phi}(\Omega)$ is then defined as the linear hull of the set $K_{\Phi}(\Omega)$. The space $L_{\Phi}(\Omega)$ is a Banach space under the following Luxemburg norm

$$
\|u\|_{\Phi}:=\inf \left\{k>0: \int_{\Omega} \Phi\left(\frac{u(x)}{k}\right) d x \leq 1\right\}
$$

or the equivalent Orlicz norm

$$
\|u\|_{L_{\Phi}}:=\sup \left\{\left|\int_{\Omega} u(x) v(x) d x\right|: v \in K_{\Phi^{*}}(\Omega), \int_{\Omega} \Phi^{*}(|v(x)|) d x \leq 1\right\} .
$$

For Orlicz spaces, the Hölder inequality reads as follows (see [22]):

$$
\int_{\Omega} u v d x \leq 2\|u\|_{L_{\Phi}(\Omega)}\|u\|_{L_{\Phi^{*}}(\Omega)} \quad \text { for all } u \in L_{\Phi}(\Omega) \text { and } v \in L_{\Phi^{*}}(\Omega) .
$$

The Orlicz-Sobolev space $W^{1} L_{\Phi}(\Omega)$ building upon $L_{\Phi}(\Omega)$ is the space defined by

$$
W^{1} L_{\Phi}(\Omega):=\left\{u \in L_{\Phi}(\Omega): \frac{\partial u}{\partial x_{i}} \in L_{\Phi}(\Omega), i=1,2, \ldots, N\right\} .
$$

and it is a Banach space with respect to the norm

$$
\|u\|_{1, \Phi}:=\|u\|_{\Phi}+\|\mid \nabla u\|_{\Phi} .
$$


Now, we introduce the Orlicz-Sobolev space $W_{0}^{1} L_{\Phi}(\Omega)$ as the closure of $C_{0}^{\infty}(\Omega)$ in $W^{1} L_{\Phi}(\Omega)$. It turns out that the space $W_{0}^{1} L_{\Phi}(\Omega)$ can be renormed by using as an equivalent norm

$$
\|u\|:=\|\mid \nabla u\|_{\Phi} .
$$

For an easier manipulation of the spaces defined above, we define the numbers

$$
\varphi_{0}:=\inf _{t>0} \frac{t \varphi(t)}{\Phi(t)} \quad \text { and } \quad \varphi^{0}:=\sup _{t>0} \frac{t \varphi(t)}{\Phi(t)} .
$$

Throughout this paper, we assume that

$$
1<\varphi_{0} \leq \frac{t \varphi(t)}{\Phi(t)} \leq \varphi^{0}<\infty, \quad t \geq 0
$$

which assures that $\Phi$ satisfies the $\Delta_{2}$-condition, i.e.,

$$
\Phi(2 t) \leq K \Phi(t), \quad \forall t \geq 0,
$$

where $K$ is a positive constant (see [21, Proposition 2.3]).

In this paper, we also need the following condition

$$
\text { the function } t \mapsto \Phi(\sqrt{t}) \text { is convex for all } t \in[0, \infty) \text {. }
$$

We notice that Orlicz-Sobolev spaces, unlike the Sobolev spaces they generalize, are in general neither separable nor reflexive. A key tool to guarantee these properties is represented by the $\Delta_{2}$-condition (1.5). Actually, condition (1.5) assures that both $L_{\Phi}(\Omega)$ and $W_{0}^{1} L_{\Phi}(\Omega)$ are separable, see [1]. Conditions (1.5) and (1.6) assure that $L_{\Phi}(\Omega)$ is a uniformly convex space and thus, a reflexive Banach space (see [21]); consequently, the Orlicz-Sobolev space $W_{0}^{1} L_{\Phi}(\Omega)$ is also a reflexive Banach space.

Proposition 1.1 (see $[4,20,21]$ ). Let $u \in W_{0}^{1} L_{\Phi}(\Omega)$. Then we have

(i) $\|u\|^{\varphi^{0}} \leq \int_{\Omega} \Phi(|\nabla u|) d x \leq\|u\|^{\varphi_{0}}$ if $\|u\|<1$.

(ii) $\|u\|^{\varphi_{0}} \leq \int_{\Omega} \Phi(|\nabla u|) d x \leq\|u\|^{\varphi^{0}}$ if $\|u\|>1$.

We also find that with the help of condition (1.4), the Orlicz-Sobolev space $W_{0}^{1} L_{\Phi}(\Omega)$ is continuously embedded in the classical Sobolev space $W_{0}^{1, \varphi_{0}}(\Omega)$, as a result, $W_{0}^{1} L_{\Phi}(\Omega)$ is continuously and compactly embedded in the classical Lebesgue space $L^{q}(\Omega)$ for all $1 \leq q<\varphi_{0}^{*}$, where $\varphi_{0}^{*}=+\infty$ if $\varphi_{0} \geq N$ and $\varphi_{0}^{*}:=\frac{N \varphi_{0}}{N-\varphi_{0}}$ if $\varphi_{0}<N$. Now, let us give some examples of functions $\varphi: \mathbb{R} \rightarrow \mathbb{R}$ which are odd, increasing homeomorphism from $\mathbb{R}$ onto $\mathbb{R}$ and satisfy conditions (1.4) and (1.6), the readers can find them in [4, 20].

\section{Example 1.2.}

(1) Let $\varphi(t)=p|t|^{p-2} t, t \in \mathbb{R}, p>1$. A simple computation shows that $\varphi_{0}=\varphi^{0}=p$. In this case, the corresponding Orlicz space $L_{\Phi}(\Omega)$ is the classical Lebesgue space $L^{p}(\Omega)$ while the Orlicz-Sobolev space $W_{0}^{1} L_{\Phi}(\Omega)$ is the classical Sobolev space $W_{0}^{1, p}(\Omega)$. Therefore, we obtain the $p$-Kirchhoff type problems as in $[2,5,12,18,19,24,25]$ and the references cited there. 
(2) Let $\varphi(t)=\log \left(1+|t|^{s}\right)|t|^{p-2} t, t \in \mathbb{R}, p, s>1$. Then we can deduce that $\varphi_{0}=p$ and $\varphi^{0}=p+s$.

(3) Let $\varphi(t)=\frac{|t|^{p-2} t}{\log (1+|t|)}$ if $t \neq 0, \varphi(0)=0$ with $p>2$. Then we can deduce that $\varphi_{0}=p-1$ and $\varphi^{0}=p$.

Definition 1.3. Let $X$ be a real Banach space. We denote by $\mathcal{W}_{X}$ the class of functional $A: X \rightarrow \mathbb{R}$ possessing the following property: if $\left\{u_{m}\right\}$ is a sequence in $X$ weakly converging to $u \in X$ and $\liminf _{m \rightarrow \infty} A\left(u_{m}\right) \leq A(u)$, then $\left\{u_{m}\right\}$ has a subsequence strongly converging to $u$.

The key in our argument is the following result which was presented in the paper by B. Ricceri [23].

Proposition 1.4 (see [23]). Let $X$ be a separable and reflexive real Banach space; $\Psi: X \rightarrow \mathbb{R}$ a coercive, sequentially weakly lower semicontinuous $C^{1}$ functional, belonging to $\mathcal{W}_{X}$, bounded on each bounded subset of $X$ and whose derivative admits a continuous inverse on $X^{*} ; J: X \rightarrow \mathbb{R}$ a $C^{1}$ functional with compact derivative. Assume that $\Phi$ has a strict local minimum $x_{0}$, with $\Psi\left(x_{0}\right)=J\left(x_{0}\right)=0$. Finally, assume that

$$
\max \left\{\limsup _{\|x\| \rightarrow+\infty} \frac{J(x)}{\Psi(x)}, \limsup _{x \rightarrow x_{0}} \frac{J(x)}{\Psi(x)}\right\} \leq 0
$$

and that

$$
\sup _{x \in X} \min \{\Psi(x), J(x)\}>0 .
$$

Set

$$
\theta^{*}:=\inf \left\{\frac{\Psi(x)}{J(x)}: x \in X, \min \{\Psi(x), J(x)\}>0\right\} .
$$

Then, for each compact interval $\Lambda \subset\left(\theta^{*},+\infty\right)$, there exists a number $\delta>0$ with the following property: for every $\lambda \in \Lambda$ and every $C^{1}$ functional $\Gamma: X \rightarrow \mathbb{R}$ with compact derivative, there exists $\mu^{*}>0$ such that for each $\mu \in\left[0, \mu^{*}\right]$, the equation

$$
\Psi^{\prime}(x)=\lambda J^{\prime}(x)+\mu \Gamma^{\prime}(x)
$$

has at least three solutions whose norms are less than $\delta$.

\section{Main results}

Let us introduce the class of nonlinearities involved in problem (1.1). If $N \geq \varphi_{0}$, let us denote by $\mathcal{A}$ the class of all Carathéodory function $f: \Omega \times \mathbb{R} \rightarrow \mathbb{R}$ such that

$$
\sup _{(x, t) \in \Omega \times \mathbb{R}} \frac{|f(x, t)|}{1+|t|^{q}}<+\infty,
$$

where $0<q<\varphi_{0}^{*}-1$, while when $N<\varphi_{0}$ let us denote by $\mathcal{A}$ the class of all Carathéodory function $f: \Omega \times \mathbb{R} \rightarrow \mathbb{R}$ such that, for each $M>0$, the function $x \mapsto \sup _{|t| \leq M}|f(x, t)|$ belongs to $L^{1}(\Omega)$. 
If $f \in \mathcal{A}$, we put

$$
F(x, t)=\int_{0}^{t} f(x, s) d s, \quad(x, t) \in \Omega \times \mathbb{R}
$$

and

$$
\widehat{M}(t)=\int_{0}^{t} M(s) d s, \quad t \geq 0 .
$$

Furthermore, let us define the functionals $\rho, \Psi, J: X=W_{0}^{1} L_{\Phi}(\Omega) \rightarrow \mathbb{R}$ by

$$
\begin{array}{ll}
\rho(u) & =\int_{\Omega} \Phi(|\nabla u|) d x, \quad \Psi(u)=\widehat{M}(\rho(u)), \\
J(u) & =\int_{\Omega} F(x, u) d x, \quad u \in X .
\end{array}
$$

Some simple computations show that the functional $\Psi$ and $J$ are of $C^{1}$ in $X$, and their derivatives are given by

$$
\begin{aligned}
\Psi^{\prime}(u)(v) & =M\left(\int_{\Omega} \Phi(|\nabla u|) d x\right) \int_{\Omega} a(|\nabla u|) \nabla u \cdot \nabla v d x \\
J^{\prime}(u)(v) & =\int_{\Omega} f(x, u) v d x
\end{aligned}
$$

for all $u, v \in X$. Moreover, since $f \in \mathcal{A}$, the mapping $J^{\prime}: X \rightarrow X^{\prime}$ is compact.

Definition 2.1. We say that $u \in X$ is a weak solution of problem (1.1) if

$$
\begin{aligned}
& M\left(\int_{\Omega} \Phi(|\nabla u|) d x\right) \int_{\Omega} a(|\nabla u|) \nabla u \cdot \nabla v d x \\
& -\lambda \int_{\Omega} f(x, u) v d x-\lambda \int_{\Omega} g(x, u) v d x=0
\end{aligned}
$$

for all $u, v \in X$.

Our main result of this paper is given by the following theorem.

Theorem 2.2. Let $f \in \mathcal{A}$ and assume the following conditions are satisfied:

$\left(M_{0}\right)$ There exist two constants $m_{0}>0$ and $1<\alpha<\frac{\varphi_{0}^{*}}{\varphi^{0}}$ such that $M(t) \geq$ $m_{0} t^{\alpha-1}$ for all $t \geq 0$, where $\varphi_{0}^{*}=+\infty$ if $\varphi_{0} \geq N$ and $\varphi_{0}^{*}=\frac{N \varphi_{0}}{N-\varphi_{0}}$ if $\varphi_{0}<N$

$\left(F_{1}\right) \sup _{u \in X} \int_{\Omega} F(x, u(x)) d x>0$;

$\left(F_{2}\right) \lim \sup _{t \rightarrow 0} \frac{\sup _{x \in \Omega} F(x, t)}{|t|^{\alpha \varphi^{0}}} \leq 0$;

$\left(F_{3}\right) \lim \sup _{|t| \rightarrow+\infty} \frac{\sup _{x \in \Omega} F(x, t)}{|t|^{\alpha \varphi_{0}}} \leq 0$.

Under such hypotheses, if we set

$$
\theta^{*}:=\inf \left\{\frac{\widehat{M}\left(\int_{\Omega} \Phi(|\nabla u|) d x\right)}{\int_{\Omega} F(x, u(x)) d x}: u \in W_{0}^{1} L_{\Phi}(\Omega), \int_{\Omega} F(x, u(x)) d x>0\right\}
$$


then for each compact interval $\Lambda \subset\left(\theta^{*},+\infty\right)$, there exists a number $\delta>0$ with the following property: for every $\lambda \in \Lambda$ and every $g \in \mathcal{A}$ there exists $\mu^{*}>0$ such that, for each $\mu \in\left[0, \mu^{*}\right]$, problem (1.1) has at least three weak solutions whose norms are less than $\delta$.

Remark 2.3. In [4], the authors consider the special case when $M(t) \equiv 1$ and assume that (see the condition $\left(a_{1}\right)$ of [4, Theorem 3.1], the same for the assumption $\left(b_{1}\right)$ of Theorem 3.2):

$$
\limsup _{|t| \rightarrow+\infty} \frac{\sup _{x \in \Omega} F(x, t)}{|t|^{\varphi^{0}}} \leq 0 .
$$

We think that the valid assumption should be

$$
\limsup _{|t| \rightarrow+\infty} \frac{\sup _{x \in \Omega} F(x, t)}{|t|^{\varphi_{0}}} \leq 0 .
$$

Indeed, the series of inequalities before relation (13) of [4] is not correct. If $\|u\|>1$, then it follows from Proposition 1.1 that

$$
\|u\|^{\varphi_{0}} \leq \rho(u) \leq\|u\|^{\varphi^{0}} .
$$

For this reason, the authors cannot use relation (11) in order to obtain (13) as in [4].

We first prove the following useful result, which helps us to apply Proposition 1.4

\section{Lemma 2.4 .}

(i) The functional $\Psi$ is sequentially weakly lower semicontinuous;

(ii) $\Psi$ belongs to the class $\mathcal{W}_{X}$.

Proof. (i) Let $\left\{u_{m}\right\} \subset X$ be a sequence that converges weakly to $u$ in $X$. Then, from the proof of $[21$, Lemma 4.3] we deduce that the functional $\rho: X \rightarrow \mathbb{R}$,

$$
u \mapsto \rho(u)=\int_{\Omega} \Phi(|\nabla u|) d x
$$

is weakly lower semi-continuous, i.e.,

$$
\int_{\Omega} \Phi(|\nabla u|) d x \leq \liminf _{m \rightarrow \infty} \int_{\Omega} \Phi\left(\left|\nabla u_{m}\right|\right) d x .
$$

Combining (2.3) with the continuity and monotonicity of the function $t \mapsto$ $\widehat{M}(t)$, we get

$$
\begin{aligned}
\liminf _{m \rightarrow \infty} \Psi\left(u_{m}\right) & =\liminf _{m \rightarrow \infty} \widehat{M}\left(\int_{\Omega} \Phi\left(\left|\nabla u_{m}\right|\right) d x\right) \\
& \geq \widehat{M}\left(\liminf _{m \rightarrow \infty} \int_{\Omega} \Phi\left(\left|\nabla u_{m}\right|\right) d x\right) \\
& \geq \widehat{M}\left(\int_{\Omega} \Phi(|\nabla u|) d x\right)
\end{aligned}
$$




$$
=\Psi(u) .
$$

Thus, the functional $\Psi$ is sequentially weakly lower semicontinuous.

(ii) Since $\widehat{M}$ is continuous and strictly increasing, it suffices to show that $\rho \in \mathcal{W}_{X}$. So, let $\left\{u_{m}\right\}$ be a sequence weakly converging to $u$ in $X$ and let $\lim \inf _{m \rightarrow \infty} \rho\left(u_{m}\right) \leq \rho(u)$. Since the functional $\rho$ is sequentially weakly lower semicontinuous, there exists a subsequence of $\left\{u_{m}\right\}$, still denoted by $\left\{u_{m}\right\}$ such that

$$
\lim _{m \rightarrow \infty} \rho\left(u_{m}\right)=\rho(u) .
$$

Since $\left\{u_{m}\right\}$ converges weakly to $u$ we also have $\left\{\frac{u_{m}+u}{2}\right\}$ converges weakly to $u$ in $X$. From (i), the functional $\Psi$ is sequentially weakly lower semicontinuous, so

$$
\liminf _{m \rightarrow \infty} \rho\left(\frac{u_{m}+u}{2}\right) \geq \rho(u) .
$$

We assume by contradiction that $\left\{u_{m}\right\}$ does not converge to $u$ in $X$. Hence, there exists $\epsilon_{0}>0$ such that $\left\|u_{m}-u\right\| \geq \epsilon_{0}$ or $\left|\frac{u_{m}-u}{2}\right| \geq \frac{\epsilon_{0}}{2}$. By Proposition 1.1 ,

$$
\rho\left(\frac{u_{m}-u}{2}\right) \geq \max \left\{\epsilon_{0}^{\varphi_{0}}, \epsilon_{0}^{\varphi^{0}}\right\} .
$$

On the other hand, by (1.5) and (1.6), we can apply [17, Lemma 2.1] in order to obtain

$$
\frac{1}{2} \rho\left(u_{m}\right)+\frac{1}{2} \rho(u)-\rho\left(\frac{u_{m}+u}{2}\right) \geq \rho\left(\frac{u_{m}-u}{2}\right) \geq \max \left\{\epsilon_{0}^{\varphi_{0}}, \epsilon_{0}^{\varphi^{0}}\right\} .
$$

It follows from (2.5) and (2.7) that

$$
\rho(u)-\max \left\{\epsilon_{0}^{\varphi_{0}}, \epsilon_{0}^{\varphi^{0}}\right\} \geq \limsup _{m \rightarrow \infty} \rho\left(\frac{u_{m}+u}{2}\right) .
$$

From (2.6) and (2.8), we obtain a contradiction. This shows that $\left\{u_{m}\right\}$ converges strongly to $u$ and the functional $\Psi$ belongs to the class $\mathcal{W}_{X}$.

Proof of Theorem 2.2. We wish to apply Proposition 1.4 taking $X=W_{0}^{1} L_{\Phi}(\Omega)$, $\Psi$ and $J$ are as before. Then $J$ is a $C^{1}$ functional with compact derivative. Moreover, by Lemma $2.4, \Psi$ is a sequentially weakly lower semicontinuous and $C^{1}$ functional belonging to $\mathcal{W}_{X}$, and a simple computation shows that it is also coercive. In fact, if $\|u\|>1$, by $\left(M_{0}\right)$ and Proposition 1.1 we have

$$
\begin{aligned}
\Psi(u) & =\widehat{M}\left(\int_{\Omega} \Phi(|\nabla u|) d x\right) \\
& \geq \frac{m_{0}}{\alpha}\left(\int_{\Omega} \Phi(|\nabla u|) d x\right)^{\alpha} \\
& \geq \frac{m_{0}}{\alpha}\|u\|^{\varphi_{0} \alpha}
\end{aligned}
$$

from which we have the coercivity of $\Psi$. It is evident that $u_{0}=0$ is the only global minimum of $\Psi$ and that $\Psi\left(u_{0}\right)=J\left(u_{0}\right)=0$. 
Moreover, it is easy to see that, if $\|u\| \leq r$, then

$$
\Psi(u) \leq \max \left\{\widehat{M}(1), \widehat{M}\left(r^{\varphi^{0}}\right)\right\}
$$

and so $\Psi$ is bounded on each bounded subset of $X$.

Now, let us show that the operator $\Psi^{\prime}: X \rightarrow X^{*}$ is invertible on $X$. On account of the well-known Minty-Browder theorem (Theorem 26.A(d) of [26]), it suffices to prove that $\Psi$ is strictly convex, hemicontinuous and coercive in the sense of monotone operators.

So, let $u, v \in X$ with $u \neq v$ and $\lambda, \mu \in[0,1]$ with $\lambda+\mu=1$. Notice that the function $\varphi$ is increasing in $\mathbb{R}$ we have for all $\xi, \psi \in \mathbb{R}^{N}$,

$$
(\varphi(|\xi|)-\varphi(|\psi|))(|\xi|-|\psi|) \geq 0
$$

with the equality if and only if $\xi=\psi$. Thus, we can deduce that

$$
(a(|\xi|)|\xi|-a(|\psi|)|\psi|)(|\xi|-|\psi|) \geq 0
$$

for all $\xi, \psi \in \mathbb{R}^{N}$, with the equality if and only if $\xi=\psi$. On the other hand, some simple computations show that

$$
(a(|\xi|) \xi-a(|\psi|) \psi) \cdot(\xi-\psi) \geq(a(|\xi|)|\xi|-a(|\psi|)|\psi|)(|\xi|-|\psi|)
$$

for all $\xi, \psi \in \mathbb{R}^{N}$. Consequently, we conclude that

$$
(a(|\xi|) \xi-a(|\psi|) \psi) \cdot(\xi-\psi) \geq 0
$$

for all $\xi, \psi \in \mathbb{R}^{N}$, with the equality if and only if $\xi=\psi$.

From the above information, the operator $\rho^{\prime}: X \rightarrow X^{*}$ given by

$$
\rho^{\prime}(u)(v)=\int_{\Omega} a(|\nabla u|) \nabla u \cdot \nabla v d x
$$

is strictly monotone, so by Proposition 25.10 of [26], $\rho$ is strictly convex. Moreover, since $M$ is nondecreasing the function $\widehat{M}$ is convex in $[0,+\infty)$. Thus, we have

$$
\widehat{M}(\rho(\lambda u+\mu v))<\widehat{M}(\lambda \rho(u)+\mu \rho(v)) \leq \lambda \widehat{M}(\rho(u))+\mu \widehat{M}(\rho(u))
$$

This shows that $\Psi$ is strictly convex and, as already said, that $\Psi^{\prime}$ is strictly monotone.

For any $u \in X$ with $\|u\|>1$, by $\left(M_{0}\right)$ and Proposition 1.1, one has

$$
\begin{aligned}
\frac{\Psi^{\prime}(u)(u)}{\|u\|} & =\frac{M(\rho(u)) \int_{\Omega} a(|\nabla u|)|\nabla u|^{2} d x}{\|u\|} \\
& \geq m_{0} \varphi_{0} \frac{(\rho(u))^{\alpha}}{\|u\|} \\
& \geq m_{0} \varphi_{0}\|u\|^{\varphi_{0} \alpha-1},
\end{aligned}
$$

from which we have the coercivity of $\Psi^{\prime}$. Standard arguments ensure that $\Psi^{\prime}$ is hemicontinuous. Thus, in view of Theorem 26.A(d) of [26] there exists $\Psi^{\prime-1}: X^{*} \rightarrow X$ and it is bounded. Let us prove that $\Psi^{\prime-1}$ is continuous by showing that it is sequentially continuous. 
Let $\left\{w_{m}\right\} \subset X^{*}$ be a sequence strongly converging to $w \in X^{*}$ and let $u_{m}=\Psi^{\prime-1}\left(w_{m}\right), m=1,2, \ldots$, and $u=\Psi^{\prime-1}(w)$. Then, $\left\{u_{m}\right\}$ is bounded in $X$ and without loss of generality, we can assume that it converges weakly to a certain $u_{0} \in X$. Since $\left\{w_{m}\right\}$ converges strongly to $w$, it is easy to see that

$$
\lim _{m \rightarrow \infty} \Psi^{\prime}\left(u_{m}\right)\left(u_{m}-u_{0}\right)=\lim _{m \rightarrow \infty} w_{m}\left(u_{m}-u_{0}\right)=0
$$

or

$$
\lim _{m \rightarrow \infty} M\left(\int_{\Omega} \Phi\left(\left|\nabla u_{m}\right|\right) d x\right) \int_{\Omega} a\left(\left|\nabla u_{m}\right|\right) \nabla u_{m} \cdot\left(\nabla u_{m}-\nabla u_{0}\right) d x=0 .
$$

Using Proposition 1.1, since $\left\{u_{m}\right\}$ is bounded in $X$, passing to a subsequence, if necessary, we may assume that

$$
\int_{\Omega} \Phi\left(\left|\nabla u_{m}\right|\right) d x \rightarrow t_{0} \geq 0 \text { as } m \rightarrow \infty .
$$

If $t_{0}=0$, then using Proposition 1.1 again, $\left\{u_{m}\right\}$ converges strongly to $u_{0}=0$ in $X$ and the proof is finished because of the continuity and injectivity of $\Psi^{\prime}$. If $t_{0}>0$, it follows from the continuity of the function $M$ that

$$
M\left(\int_{\Omega} \Phi\left(\left|\nabla u_{m}\right|\right) d x\right) \rightarrow M\left(t_{0}\right) \text { as } m \rightarrow \infty .
$$

Thus, by $\left(M_{0}\right)$, for sufficiently large $m$, we have

$$
M\left(\int_{\Omega} \Phi\left(\left|\nabla u_{m}\right|\right) d x\right) \geq C_{4}>0 .
$$

From (2.10), (2.11), it follows that

$$
\lim _{m \rightarrow \infty} \int_{\Omega} a\left(\left|\nabla u_{m}\right|\right) \nabla u_{m} \cdot\left(\nabla u_{m}-\nabla u_{0}\right) d x=0 .
$$

From (2.12) and the fact that $\left\{u_{m}\right\}$ converges weakly to $u_{0}$ in $X$ we can apply Lemma 5 of [20] in order to deduce that $\left\{u_{m}\right\}$ converges strongly to $u_{0}$ in $X$. The continuity and injectivity of $\Psi^{\prime}$ imply that $\left\{u_{m}\right\}$ converges strongly to $u$, so $\Psi^{\prime-1}$ is continuous.

Now, we want to define a number $r$ such that $\alpha \varphi^{0}<r<\varphi_{0}^{*}$ and $r>q+1$. For this purpose, if $N \leq \varphi_{0}$, then $\varphi_{0}^{*}=+\infty$ and it suffices to choose $r:=$ $\max \left\{\alpha \varphi^{0}+1, q+1\right\}$. On the other hand, if $N>\varphi_{0}$, from the assumption $(M)$ if follows easily that $\alpha \varphi^{0}<\frac{N \varphi_{0}}{N-\varphi_{0}}$. So, if we choose a number $\nu>0$ such that $\alpha \varphi^{0}+\nu<\frac{N \varphi_{0}}{N-\varphi_{0}}$, then the number $r$ is defined by $r:=\max \left\{\alpha \varphi^{0}+\nu, q+1\right\}$, which satisfies all the requirements. Since $f \in \mathcal{A}$ we can easily get that

$$
\sup _{(x, t) \in \Omega \times \mathbb{R}} \frac{|F(x, t)|}{1+|t|^{r}}<+\infty .
$$

Next, fix $\epsilon>0$. In the sequel, $C_{i}$ will denote positive constants independent of $\epsilon$ and $u \in X$. Now, from the assumption $\left(F_{2}\right)$, there exists $\eta_{1}>0$ such that

$$
|F(x, t)| \leq \epsilon|t|^{\alpha \varphi^{0}}
$$


for each $x \in \Omega$ and $|t| \in\left[0, \eta_{1}\right]$.

From (2.13), it follows that, for a suitable positive constant $C_{1}$ that

$$
|F(x, t)| \leq C_{1}|t|^{r}
$$

for each $(x, t) \in \Omega \times\left(\mathbb{R} \backslash\left[-\eta_{1}, \eta_{1}\right]\right)$. Consequently, from (2.14) and (2.15) we have

$$
|F(x, t)| \leq \epsilon|t|^{\alpha \varphi^{0}}+C_{1}|t|^{r}
$$

for each $(x, t) \in \Omega \times \mathbb{R}$. So by the compact embeddings of $X$ in $L^{\alpha \varphi^{0}}(\Omega)$ and $L^{r}(\Omega)$, for some positive constants $C_{2}, C_{3}$, one has for all $u \in X$ with $\|u\|<1$,

$$
\begin{aligned}
J(u) & \leq \epsilon\|u\|_{L^{\alpha \varphi^{0}}(\Omega)}^{\alpha \varphi^{0}}+C_{1}\|u\|_{L^{r}(\Omega)}^{r} \\
& \leq \epsilon C_{2}\|u\|^{\alpha \varphi^{0}}+C_{3}\|u\|^{r} \\
& \leq \epsilon C_{2}(\rho(u))^{\alpha}+C_{3}(\rho(u))^{\frac{r}{\varphi^{0}}} \\
& \leq \epsilon C_{2} \frac{\alpha}{m_{0}} \Psi(u)+C_{3}\left(\frac{\alpha}{m_{0}} \Psi(u)\right)^{\frac{r}{\alpha \varphi^{0}}}
\end{aligned}
$$

Consequently, since $r>\alpha \varphi^{0}$ and the fact that $\Psi(u) \rightarrow 0$ as $u \rightarrow 0$ we get

$$
\limsup _{u \rightarrow 0} \frac{J(u)}{\Psi(u)} \leq \epsilon C_{2} \frac{\alpha}{m_{0}} .
$$

By $\left(F_{3}\right)$, for all $\epsilon>0$, there exists $\eta_{2}>0$ such that

$$
|F(x, t)| \leq \epsilon|t|^{\alpha p_{0}}
$$

for all $x \in \Omega$ and $|t|>\eta_{2}$.

Now, for $\|u\|>1$ large enough, from (2.19) and Proposition 1.1 we have

$$
\begin{aligned}
\frac{J(u)}{\Psi(u)} & =\frac{J(u)}{\widehat{M}(\rho(u))} \\
& \leq \frac{\int_{\left\{x \in \Omega:|u(x)| \leq \eta_{2}\right\}} F(x, u) d x}{\frac{m_{0}}{\alpha}\|u\|^{\alpha \varphi_{0}}}+\frac{\int_{\left\{x \in \Omega:|u(x)|>\eta_{2}\right\}} F(x, u) d x}{\frac{m_{0}}{\alpha}\|u\|^{\alpha \varphi_{0}}} \\
& \leq \frac{|\Omega| \sup _{(x, t) \in \Omega \times\left[-\eta_{2}, \eta_{2}\right]} F(x, t)}{\frac{m_{0}}{\alpha}\|u\|^{\alpha \varphi_{0}}}+\frac{\epsilon u \|_{L^{\alpha \varphi_{0}}(\Omega)}^{\alpha \varphi_{0}}}{\frac{m_{0}}{\alpha}\|u\|^{\alpha \varphi_{0}}} \\
& \leq \frac{|\Omega| \sup _{(x, t) \in \Omega \times\left[-\eta_{2}, \eta_{2}\right]} F(x, t)}{\frac{m_{0}}{\alpha}\|u\|^{\alpha \varphi_{0}}}+C_{4} \epsilon .
\end{aligned}
$$

From (2.20) we have

$$
\limsup _{\|u\| \rightarrow+\infty} \frac{J(u)}{\Psi(u)} \leq C_{4} \epsilon \text {. }
$$

Since $\epsilon>0$ is arbitrary, relations (2.18) and (2.21) imply that

$$
\max \left\{\limsup _{u \rightarrow 0} \frac{J(u)}{\Psi(u)}, \limsup _{\|u\| \rightarrow+\infty} \frac{J(u)}{\Psi(u)}\right\} \leq 0 .
$$


Hence, all assumptions of Proposition 1.4 are satisfied. So, for each compact interval $\Lambda \subset\left(\theta^{*},+\infty\right)$, there exists a number $\delta>0$ with the property described in the conclusion of Proposition 1.4. Fix $\lambda \in \Lambda$ and $g \in \mathcal{A}$. Put

$$
\Gamma(u)=\int_{\Omega} G(x, u) d x, \quad G(x, t)=\int_{0}^{t} g(x, s) d s
$$

for all $u \in X$. Then, $\Gamma$ is a $C^{1}$ functional on $X$ with compact derivative. So, there exists $\mu^{*}>0$ such that, for each $\mu \in\left[0, \mu^{*}\right]$, the equation

$$
\Psi^{\prime}(u)=\lambda J^{\prime}(u)+\mu \Gamma^{\prime}(u)
$$

has at least three solutions in $X$ whose norms are less than $\delta$. But the solutions in $X$ of the above equation are exactly the weak solutions of problem (1.1) and thus, the proof of Theorem 2.2 is complete.

Acknowledgments. The author would like to thank the referees for their suggestions and helpful comments which improved the presentation of the original manuscript. This paper was done when the author was working at the Division of Mathematical Sciences, School of Physical and Mathematical Sciences, Nanyang Technological University, Singapore, as a Research Fellow.

\section{References}

[1] R. Adams, Sobolev Spaces, Academic Press, New York, 1975.

[2] A. Bensedik and M. Bouchekif, On an elliptic equation of Kirchhoff-type with a potential asymptotically linear at infinity, Math. Comput. Modelling 49 (2009), no, 5-6, 10891096.

[3] F. Cammaroto and L. Vilasi, Multiple solutions for a Kirchhoff-type problem involving the $p(x)$-Laplacian operator, Nonlinear Anal. 74 (2011), no. 5, 1841-1852.

[4] F. Cammaroto and L. Vilasi, Multiple solutions for a nonhomogeneous Dirichlet problem in Orlicz-Sobolev spaces, Appl. Math. Comput. 218 (2012), no. 23, 11518-11527.

[5] C. Y. Chen, Y. C. Kuo, and T. F. Wu, The Nehari manifold for a Kirchhoff type problem involving sign-changing weight functions, J. Differential Equations 250 (2011), no. 4, 1876-1908.

[6] M. Chipot and B. Lovat, Some remarks on nonlocal elliptic and parabolic problems, Nonlinear Anal. 30 (1997), no. 7, 4619-4627.

[7] N. T. Chung, Multiple solutions for a $p(x)$-Kirchhoff-type equation with sign-changing nonlinearities, Complex Variables and Elliptic Equations, 2012, 1-10, iFirst.

[8] _ Multiplicity results for a class of $p(x)$-Kirchhoff type equations with combined nonlinearities, Electron. J. Qual. Theory Differ. Equ. 2012 (2012), no. 42, 1-13.

[9] Ph. Clément, M. Garciá-Huidobro, R. Manásevich, and K. Schmitt, Mountain pass type solutions for quasilinear elliptic equations, Calc. Var. Partial Differential Equations 11 (2000), no. 1, 33-62.

[10] Ph. Clément, B. dePagter, G. Sweers, and F. deTh?in, Existence of solutions to a semilinear elliptic system through Orlicz-Sobolev spaces, Mediterr. J. Math. 1 (2004), no. $3,241-267$.

[11] F. Colasuonno and P. Pucci, Multiplicity of solutions for $p(x)$-polyharmonic elliptic Kirchhoff equations, Nonlinear Anal. 74 (2011), no. 17, 5962-5974.

[12] F. J. S. A. Corrêa and G. M. Figueiredo, On a p-Kirchhoff equation via Krasnoselskii's genus, Appl. Math. Letters 22 (2009), no. 6, 819-822. 
[13] G. Dai and R. Hao, Existence of solutions for a $p(x)$-Kirchhoff-type equation, J. Math. Anal. Appl. 359 (2009), no. 1, 275-284

[14] X. L. Fan, On nonlocal p(x)-Laplacian Dirichlet problems, Nonlinear Anal. 72 (2010), no. 7-8, 3314-3323.

[15] F. Fang and Z. Tan, Existence and Multiplicity of solutions for a class of quasilinear elliptic equations: An Orlicz-Sobolev setting, J. Math. Anal. Appl. 389 (2012), no. 1, 420-428.

[16] G. Kirchhoff, Mechanik, Teubner, Leipzig, Germany, 1883.

[17] J. Lamperti, On the isometries of certain function-spaces, Pacific J. Math. 8 (1958), 459-466.

[18] D. Liu, On a p-Kirchhoff equation via fountain theorem and dual fountain theorem, Nonlinear Anal. 72 (2010), no. 1, 302-308.

[19] T. F. Ma, Remarks on an elliptic equation of Kirchhoff type, Nonlinear Anal. 63 (2005), 1967-1977.

[20] M. Mihăilescu and D. Repovš, Multiple solutions for a nonlinear and non-homogeneous problem in Orlicz-Sobolev spaces, Appl. Math. Comput. 217 (2011), 6624-6632.

[21] M. Mihăilescu and V. Rădulescu, Neumann problems associated to non-homogeneous differential operators in Orlicz-Sobolev spaces, Ann. Inst. Fourier 6 (2008), no. 6, 20872111.

[22] M. M. Rao and Z. D. Ren, Theory of Orlicz Spaces, Marcel Dekker Inc., New York, 1991.

[23] B. Ricceri, A further three critical points theorem, Nonlinear Anal. 71 (2009), no. 9, 4151-4157.

[24] - On an elliptic Kirchhoff-type problem depending on two parameters, J. Global Optim. 46 (2010), no. 4, 543-549.

[25] J. J. Sun and C. L. Tang, Existence and multiplicity of solutions for Kirchhoff type equations, Nonlinear Anal. 74 (2011), no. 4, 1212-1222.

[26] E. Zeidler, Nonlinear Functional Analysis and Applications, In Nonlinear monotone operators, Vol. II/B, Springer-Verlag, New York, 1990.

Department of Science Management \& International Cooperation QUANG BINH UNIVERSITY

312 Ly Thuong Kiet, Dong Hoi, Quang Binh, Vietnam

E-mail address: ntchung82@yahoo.com 